\title{
Introduction. Le rôle des enseignants
}

\section{Dominique Raulin}

\section{OpenEdition}

\section{Journals}

Édition électronique

URL : https://journals.openedition.org/ries/1828

DOI : $10.4000 /$ ries. 1828

ISSN : 2261-4265

\section{Éditeur}

France Education international

\section{Édition imprimée}

Date de publication : 1 septembre 2002

Pagination : 21-26

ISBN : 978-285420-554-1

ISSN : 1254-4590

\section{Référence électronique}

Dominique Raulin, "Introduction. Le rôle des enseignants ", Revue internationale d'éducation de Sèvres [En ligne], 30 | septembre 2002, mis en ligne le 24 novembre 2011, consulté le 05 juillet 2021. URL : http://journals.openedition.org/ries/1828; DOI : https://doi.org/10.4000/ries.1828 


\section{Le métier d'enseignant en Europe}

\section{Introduction}

\section{Le rôle des enseignants}

\section{Dominique Raulin}

Face aux problèmes économiques et aux questions de société, on se tourne volontiers vers l'École pour chercher des réponses et trouver des solutions. L'École occupe ainsi une place centrale dans tous les pays démocratiques. Pour renforcer cette situation unique, gardons à l'esprit que tout le monde est allé à l'école, a une expérience de l'enseignement et a donc un point de vue personnel sur les bonnes méthodes, sur la meilleure pédagogie ou sur les contenus essentiels! Aucune autre institution ne peut se prévaloir, depuis le milieu du XX ${ }^{\mathrm{e}}$ siècle, d'une telle familiarité de la part des habitants de tous âges d'un pays.

Peut-être à cause de cette connaissance largement partagée, l'École en France est souvent absente du débat politique ou des travaux parlementaires. Treize ans après, on cite encore comme référence la loi d'orientation du 10 juillet 1989 qui "place l'élève au centre du système». Non seulement, les débats sont rares, mais en plus ils sont souvent faussés par des références idéologiques ${ }^{1}$ : école de masse ou élitisme républicain? connaissances ou compétences? pédagogie de l'exemple ou pédagogie de l'expérience? béhaviorisme ou constructivisme? intégration ou ségrégation?...

Toutes ces questions portent sur le fonctionnement et l'organisation des classes et des établissements, faisant abstraction de celles et ceux qui implicitement ou parfois explicitement ont à donner des réponses aux élèves, comme si les enseignants étaient les applicateurs neutres d'une réglementation.

Manuela Esteves montre avec clarté qu'il n'en est pas ainsi, en citant l'échec de la réforme des curricula au Portugal (1989-1994), due entre autres à «l'éloignement des enseignants d'une participation effective aux décisions qui ont été prises». Parlant de la prise en compte des réformes en Espagne, Juan Carlos Gonzalez Faraco affirme: «Une majorité, peut-être plus silencieuse, ne se sentait pas concernée [par les réformes] : la réforme émane des bureaux officiels, elle n'a pas pris en compte le corps enseignant et c'est un échec dans la pratique».

1. En France, on peut y voir encore une des conséquences des événements de mai 1968. 
Un deuxième indice de la reconnaissance implicite du rôle particulier et personnel que joue chaque enseignant est le développement de nouvelles procédures de recours offertes aux parents: Christian Maroy explique qu'en Communauté française de Belgique ( $\mathrm{CFB}$ ), un décret vient d'être pris qui «renforce les droits et l'égalité des élèves en organisant un contrôle plus strict des décisions des enseignants ou des établissements, notamment dans les conseils de classe». Cette possibilité est interprétée comme la marque de la subjectivité des décisions prises par les enseignants.

Troisième indice, la généralisation de structures de régulation auxquelles sont associés les différents partenaires concernant les choix généraux dans un établissement ou un réseau d'établissements : Marylin Osborn rappelle qu'en Angleterre "l'école primaire [a été transformée] en un lieu d'enseignement de plus en plus encadré qui doit se plier à des exigences et à des pressions externes». Cette évolution est loin d'être appréciée par les enseignants, comme l'indique Juan Carlos Gonzalez Faraco (Espagne): «beaucoup d'enseignants refusent ouvertement la participation des parents dans la gestion de l'école, car ils craignent une invasion de leur espace professionnel par des profanes».

\section{L'INNOVATION}

C'est donc unanime, les changements de structures, de programmes etc. ne peuvent se faire sans la participation active et "volontaire» des enseignants. Les changements pédagogiques relèvent encore plus de la (bonne) volonté des professeurs. On a donné souvent l'image d'enseignants globalement conservateurs, attachés à une image ancienne du métier et, aux dires de certains, inadaptée dorénavant aux conditions générales des établissements scolaires : en Espagne, un des présupposés de la réforme actuelle présente le professeur comme «un fonctionnaire qui s'accroche à ses privilèges et à ses habitudes, qui rejette les innovations...». Le propos peut paraître sévère et la réalité est sans doute moins uniforme que cela. En France, alors que les différentes institutions scolaires tardaient à prendre en compte des évolutions de la société, des professeurs ici ou là ont mis en place des modalités d'enseignement un peu différentes, en s'inspirant souvent des grands pédagogues ${ }^{2}$ du siècle dernier. Devant le succès apparent remporté par certains d'entre eux, la «bonne pédagogie» est devenue synonyme de "pédagogie innovante»; les bons professeurs sont ceux qui changent leurs méthodes, se remettent en cause, font évoluer leur rôle... C'est ainsi qu'en France on a créé depuis le milieu des années 1980 un Plan national d'innovations, des fonds d'aide à l'innovation, le réseau administratif innovation-valorisation des réussites... Or il faut bien constater une grande stabilité des méthodes utilisées par les enseignants. L'intégration des techno-

2. Freinet, Motessori, Vigodsky... 
logies de l'information et de la communication dans les pratiques professionnelles des enseignants en classe et en dehors de la classe, en est un exemple assez parlant. Danièle Périsset-Bagnoud explique cette inertie aux changements et à l'innovation : "L'innovation pédagogique ne se décrète pas ni ne peut se transmettre en formation initiale sans risque de déconstruction ultérieure en raison, selon l'expression de Gather Thurler (2000), de la contre-socialisation subie au contact des collègues déjà en place». Agnès van Zanten reprend le même argument en affirmant que «les enquêtes montrent que la conversion s'opère assez vite, les représentations et les pratiques déclarées des nouveaux venus se rapprochant de celles d'autres collègues du même établissement au bout de trois ou quatre ans».

Sur le plan des institutions, ce discours et ces décisions concernant l'innovation pédagogique se sont appuyés sur une nouvelle confiance accordée aux acteurs de terrain seuls susceptibles, selon certains, de trouver les réponses appropriées aux questions qui se posent localement. On pourrait y voir une reconnaissance effective des compétences des enseignants si ce transfert de responsabilité n'était accompagné d'une multiplication de systèmes d'aides et de contrôles élaborés au niveau central. Christian Maroy (CFB) cite à cet égard l'exemple des «batteries d'épreuves d'évaluation, en principe indicatives et visant à aider les enseignants dans leurs pratiques d'évaluation [...], mais ceuxci sont incités à s'y conformer de façon préventive pour se prémunir contre d'éventuels recours".

Ainsi, le discours politique incite le professeur à innover, à prendre plus d'autonomie et de responsabilité et met en œuvre parallèlement des systèmes d'évaluation et de contrôle renforcés.

\section{LE PRATICIEN RÉfLEXIF}

Le discours sur le métier reste assez théorique si l'on ne rentre pas dans le cour de celui-ci, c'est-à-dire dans la mise en œuvre des contenus que les élèves doivent acquérir.

Or, en plus des changements de société maintes fois évoqués pour expliquer l'évolution du métier d'enseignant, on découvre en étudiant les contenus d'enseignement une mutation fondamentale dans la plupart des pays occidentaux : leur présentation sous forme de compétences à développer plus que de connaissances à acquérir. Manuela Esteves signale par exemple qu'au Portugal «au cour des réformes actuelles, on trouve l'idée de développement de compétences générales...; la notion de compétence a été définie comme le savoir en usage ou en action pour inclure et dépasser les notions de connaissances...»; Christian Maroy (CFB) parle de logique de "compétences» et rappelle la mise en place «des socles de compétences et de compétences terminales à différents moments de la scolarité des élèves». 
Cette évolution majeure est la traduction scolaire de la théorie du constructivisme ${ }^{3}$. Outre la légitimité scientifique de celle-ci qu'il n'y a pas lieu de discuter ici, notons qu'elle est bien accueillie par les élèves et les parents : en effet, elle devrait permettre de reconnaître et de valoriser différentes formes d'intelligence, elle devrait encourager les pratiques de pédagogie active et de pédagogie différenciée ${ }^{4}$.

Mais ne nous y trompons pas : cette mutation n'est pas un simple artifice de présentation, comme certains ont cru pouvoir le dire. Non, il s'agit bien d'une révolution qui impose aux professeurs de changer de posture intellectuelle et de reconsidérer leurs rôles et leurs places en classe : d'une situation où, détenteurs d'un savoir, il leur «suffisait» de le présenter aux élèves, ils deviennent concepteurs et organisateurs de scénarios d'apprentissage qui permettent aux élèves de développer eux-mêmes leurs compétences, en partant du postulat qu'un savoir n'est acquis que s'il est construit.

Dès lors, on peut imaginer l'extrême difficulté que peuvent rencontrer les enseignants pour s'adapter à ce nouveau contexte : jusqu'à récemment, les instruments d'évaluation étaient rares et peu fiables, les supports didactiques peu nombreux. Les professeurs disposaient de très peu d'outils pour mettre en œuvre cette décision administrative, mais ce n'est sans doute pas cela le plus délicat. En effet, cette mutation a transformé le modèle de professionnalité en privilégiant dorénavant celui du «praticien réflexif» (cf. Christian Maroy, CFB). Cette étape est la dernière en date de l'évolution d'un modèle théorique d'enseignant «applicateur de décisions administratives», dont on a montré la fragilité, vers un modèle d'enseignant «responsable et professionnel». Il devient spécialiste des apprentissages, il prend de la distance par rapport aux situations de classe, il sait mettre en place une pédagogie active et différenciée.

Les freins et les réticences sont nombreux pour passer des intentions à la réalité dans les classes : resurgissent en priorité les oppositions idéologiques entre éducation et instruction, entre pédagogie de l'exemple et pédagogie de l'action et ensuite les décalages avec les représentations personnelles que les enseignants ont de leur métier. Agnès van Zanten rappelle qu'une étude faite auprès de jeunes professeurs de l'enseignement secondaire en France indique que la raison la plus souvent citée pour expliquer le choix de l'enseignement est «l'intérêt pour la transmission des connaissances». Ces jeunes recrutés peuvent-ils vraiment s'inscrire dans le modèle du "praticien réflexif» en privilégiant des méthodes actives?

Alors, pour que cette réforme, si unanimement promue dans les différents pays qu'on peut difficilement douter de sa pertinence, se concrétise, une réponse immédiate vient à l'esprit du naîf : il suffit de former les enseignants.

3. École de Palo Alto (1959).

4. En France, elle est dans la logique de la loi d'orientation de juillet 1989. 
En dépit de la diversité des organisations administratives, du statut des formateurs, les différents auteurs expriment assez unanimement leurs doutes quant à l'efficacité de la formation en ces domaines. Avant même la formation, il est clair que les modalités et les critères de recrutement sont modélisateurs. Agnès van Zanten développe cette idée en rappelant qu'en France, «sont défendus à travers les épreuves théoriques du CAPES et de l'agrégation, une vision de la compétence fondée sur la possession d'une culture générale se référant à des valeurs liées à la connaissance, à la transmission du patrimoine culturel et à l'idée d'un progrès technique et scientifique civilisateur». Les enseignants recrutés sur de tels critères sont-ils prêts et seront-ils réceptifs à une formation pour devenir des «praticiens réflexifs»? Après le recrutement ou l'habilitation à enseigner, dans tous les pays, on assiste à une tentative de renforcement de la professionnalisation de la formation initiale, notamment par le développement de stages en situation. À l'image des compétences à développer chez les élèves, des référentiels de métier sont rédigés pour les enseignants, par exemple en Communauté française de Belgique ou en France. Agnès van Zanten explique, par exemple, que «les auteurs des textes fondateurs des Instituts universitaires de formation des maîtres (IUFM) ont insisté, à travers la notion de référentiels de compétences, sur quatre dimensions nouvelles ou anciennes de la professionnalité enseignante».

Pour les enseignants déjà en poste depuis plusieurs années, devenir un praticien réflexif est difficile puisque c'est mettre en œuvre des méthodes et des comportements radicalement différents de leurs habitudes et de ce qu'on leur a demandé de faire pendant de nombreuses années. Cela se traduit par une formidable inertie au changement. En se référant à $\mathrm{Ph}$. Perrenoud ${ }^{5}$, Juan Carlos Gonzalez Faraco (Espagne) explique que «l'enseignant déploie plusieurs formes de résistance, explicitement ou implicitement, pour réorienter la réforme vers son propre territoire corporatif et sa culture empirique».

Danièle Périsset-Bagnoud (Suisse) cite également $\mathrm{Ph}$. Perrenoud pour montrer que la tentative actuelle de faire passer cette innovation par la formation est vouée à l'échec : "la synchronisation entre réformes scolaires et rénovations de la formation des enseignants, malgré l'apparentement intellectuel des deux propositions, reste difficile, introuvable, d'ordre mythique. L'évolution des pratiques renvoie à l'acte quotidien de l'enseignement et appartient au curriculum réel; celle des contenus, quant à elle, chevauche l'injonction institutionnelle et les choix professionnels».

La formation continue ne semble pas, sauf peut-être en Angleterre ${ }^{6}$, en mesure de faire comprendre et donc accepter les choix fondamentaux qui sous-

5. Professeur de sciences de l'éducation à l'université de Genève.

6. M. Osborn : «Ces réformes comprenaient l'introduction de nouveaux modes de gestion des établissements, de nouveaux modes d'évaluation du travail des enseignants et l'apparition de la notion de marché dans le secteur éducatif ». 
tendent cette mutation du métier. Plusieurs l'expliquent en montrant la dualité des objectifs : elle vise à "convertir» les enseignants au nouveau modèle (Christian Maroy) et non pas à les doter des outils intellectuels et matériels du changement; elle s'organise dans une stratégie plus «d'évangélisation que de professionnalisation» (Juan Carlos Gonzalez Faraco). Elle est ressentie comme «insuffisante pour préparer et soutenir les changements à introduire» (Manuela Esteves, Portugal). Finalement, la formation continue apparaît de plus en plus comme un outil de pilotage et non pas un outil de professionnalisation.

En guise de conclusion, à la lecture des différents articles, on peut constater, au-delà des différences d'organisations administratives, une évolution du métier qui va vers plus de professionnalité et plus de technicité. On ne peut assurément que se réjouir de voir «l'empirisme pédagogique» reculer au profit d'une certaine «science de l'enseignement». Mais les auteurs des différents articles insistent aussi sur une évolution simultanée de rationalisation et de contrôle assurée tant par les organes administratifs que par les partenaires de l'École, comme par exemple les procédures d'évaluation externe, la standardisation croissante des contenus d'enseignement, la concurrence entre les établissements.

Si l'on en croit Juan Carlos Gonzalez Faraco (Espagne), cette dualité aboutirait à une ambiguïté dans les décisions et les discours visant à définir le nouvel enseignant qui serait dans l'avenir un être hybride entre l'enseignant d'hier qui exerçait par vocation et était complètement voué à sa mission et celui de demain qui serait un opérateur spécialisé dans la fabrication d'une éducation économiquement efficace, au sein d'un marché concurrentiel.

En nous inspirant de la conclusion de Marylin Osborn (Angleterre), cela nous incite à poser deux questions : est-ce ce système d'éducation que nous souhaitons voir se développer? est-ce ce professeur là qui sera capable de former le futur citoyen de demain que nous voulons? 